\title{
Monotonic entropy growth for a nonlinear model of random exchanges
}

\author{
S. M. Apenko \\ I E Tamm Theory Department, P N Lebedev Physical Institute, Moscow, 119991, Russid*
}

\begin{abstract}
We present a proof of the monotonic entropy growth for a nonlinear discrete-time model of a random market. This model, based on binary collisions, also may be viewed as a particular case of Ulam's redistribution of energy problem. We represent each step of this dynamics as a combination of two processes. The first one is a linear energy-conserving evolution of the two-particle distribution, for which the entropy growth can be easily verified. The original nonlinear process is actually a result of a specific 'coarse-graining' of this linear evolution, when after the collision one variable is integrated away. This coarse-graining is of the same type as the real space renormalization group transformation and leads to an additional entropy growth. The combination of these two factors produces the required result which is obtained only by means of information theory inequalities.
\end{abstract}

PACS numbers: 05.20.Dd, 89.65.Gh, 89.70.Cf

It is widely known that for a stochastic Markov process described by a linear master equation for a distribution function $p(x, t)$ defined on some space of $x$ 's there exists a Lyapunov function which monotonically decreases while we approach equilibrium. This function is just the relative entropy $K=\sum_{x} p(x, t) \ln p(x, t) / p_{0}(x)$, where $p_{0}(x)$ is an equilibrium distribution [1] (see also earlier works by Schlögl [2] and, e.g., [3] for more recent related studies). This relative entropy has the meaning of the 'information gain' that we obtain when the knowledge about the true distribution $p(x, t)$ becomes available if we $a$ priory knew only the equilibrium one $p_{0}(x)$. $K$ may be also viewed as a total entropy production that takes place during the whole relaxation process [1 $[3]$.

There is no such general result for nonlinear evolution equations, where monotonicity of possible Lyapunov functions should be proved independently for each problem. There are, however, situations when it is just the Boltzmann entropy which monotonically grows when we approach equilibrium, the most known example being the Boltzmann equation itself where this monotonicity is established by the famous $H$-theorem [4].

Quite recently an interesting nonlinear evolution was proposed and analyzed as a gas-like economic model in a series of papers [5]. This is a discrete-time evolution for distributions $p(x)$ with continuous $x \geq 0$ and on each step of iterative procedure $p(x) \rightarrow p^{\prime}(x)$, where

$$
p^{\prime}(x)=\int_{0}^{\infty} \int_{0}^{\infty} d u d v \frac{\theta(u+v-x)}{u+v} p(u) p(v)
$$

and the $\theta$-function ensures that $u+v>x$. This model assumes that economic transactions occur by binary 'collisions' between agents who exchange money in the same way as particles in a gas exchange their energy [6] and after each collision the total amount of money they both possessed is distributed between them absolutely at random. For initial distributions with finite mean 'energy'

*Electronic address: apenko@lpi.ru $\langle x\rangle$ this process converges to exponential equilibrium distribution $p_{0}=\alpha \exp (-\alpha x)$, where $1 / \alpha=\langle x\rangle[5]$.

The structure of Eq. (11) is very transparent: We first randomly choose two values $u$ and $v$ with probability $p(u) p(v)$, then multiply it by a transition probability $W(u, v \rightarrow x)$, given here by $W(x)=1 /(u+v)$ for $0<x<u+v$, and finally sum over all possible choices of $u, v$ to obtain the new distribution $p(x)$. Thus for uniform probability density $W(x)$ the factor $1 /(u+v)$ in Eq. (11) arises simply from the normalization condition $\int W(x) d x=1$. It is easy to prove also that $\langle x\rangle$ is conserved under this nonlinear transformation [5].

In fact, this process is an example of what is known as Ulam's redistribution of energy problem, stated as follows: "Consider a vast number of particles and let us redistribute the energy of these particles... First, pair the particles at random. Second, for each pair, redistribute the total energy of the pair between these particles according to some given fixed probability law of redistribution..." 7]. Ulam believed that the distribution of energy would then converge to some final distribution independent of the initial one and later his conjecture was indeed proved in [7]. For uniform redistribution law this process is essentially the same as the money exchanges described by Eq. (1). However, the nonlinear transformation (1) first introduced in [5] in an economic context seems more suited for our study than the equation for the moments of $p(x)$ used in [7].

Since the process (10) is very similar in spirit to the evolution that leads to the Boltzmann equation we expect that the entropy $S(p)=-\int d x p(x) \ln p(x)$ should monotonically increase under this transformation. While this conjecture was first formulated already in [5], the analytical proof of this growth seems to be still lacking, mainly because standard methods do not directly work for discrete-time evolution. Note, that since entropy is obviously maximized by the exponential distribution $p_{0} \sim \exp (-\alpha x)$ under the constraint $\langle x\rangle=$ const (see e.g. [8]) it is monotonicity that has to be proved.

It should be noted here that for non-uniform redistribution laws in Ulam's problem we do not expect that entropy always grows. Indeed, in the general case the 
limiting distribution is no longer exponential [7], hence the entropy is not maximal in equilibrium. A simple example is a special law when the total energy of colliding particles is shared equally among them. In this case all particles will have the same energy in equilibrium [7] and the entropy definitely gets lower during relaxation. For this reason here we consider only uniform redistribution described by random market model of Eq. (11).

One can, of course, try to rewrite Eq. (1) in a form similar to Markov chain evolution

$$
p^{\prime}(x)=\int_{0}^{\infty} d u \mathrm{P}(x, u ; p) p(u),
$$

where 'transition probability'

$$
\mathrm{P}(x, u ; p)=\int_{0}^{\infty} d v \frac{\theta(u+v-x)}{u+v} p(v)
$$

itself depends on $p(x)$. Stochastic processes that may be related to such equations are now sometimes called nonlinear Markov processes [9] though this terminology was criticized in 10]. Regardless of what we call it, if we substitute some solution $p(x)$ of Eq. (1D) into $\mathrm{P}(x, u ; p)$ we will end with the linear equation (2) but with time dependent transition probabilities. Close to equilibrium we may take $\mathrm{P} \simeq \mathrm{P}\left(x, u ; p_{0}\right)$ which now satisfies detailed balance condition $\mathrm{P}\left(x, u ; p_{0}\right) / \mathrm{P}\left(u, x ; p_{0}\right) \sim \exp (-\alpha x+\alpha u)$ and hence will definitely lead to the monotonic entropy growth. But far from equilibrium this approach seems to be of little help.

For this reason in this note we will give a proof of the monotonic entropy growth for Eq. (1) within quite a different approach, which is based almost entirely on known information theory inequalities and utilizes the fact that certain coarse-graining transformations always result in the entropy growth.

The main idea of the proof is (i) to introduce an auxiliary linear evolution, defined on a larger space of two variables, for which entropy growth can be easily proved and then (ii) to show that (1D) is actually a result of a certain coarse-graining of this linear evolution.

For this purpose let us introduce a 'two-particle' distribution function $f(x, y)$ which after one step of evolution transforms into $f^{\prime}(x, y)$,

$$
f^{\prime}(x, y)=\int_{0}^{1} d \xi f(\xi(x+y),(1-\xi)(x+y)) .
$$

This is obviously a linear transformation and it is easy to see that it conserves positivity of $f(x, y)$, its norm and the mean 'energy' $\langle x+y\rangle$.

The physical meaning of Eq. (44) is rather clear since $f(x, y)$ describes pairs of particles. Particles, which after the collision have energies $x$ and $y$, before the collision might have any energies $u$ and $v$ provided $u+v=x+y$ i.e. we may take $u=\xi(x+y)$ and $v=(1-\xi)(x+y)$, where $0<\xi<1$ denotes a fraction of the total energy that the first particle had. Then Eq. (44) is just the sum over all possibilities (all of them having equal probabilities) that result in the values $x$ and $y$.

It should be noted, however, that (4) alone does not describe correctly evolution of the two-particle probability distribution in Ulam's problem. It takes into account only collisions within fixed pairs of particles and if we choose initial distribution as a $\delta$-function localized at some point $\left(x_{0}, y_{0}\right)$ then after the first iteration it will be uniformly smeared along the isoenergetic line $x+y=x_{0}+y_{0}$ and will not change afterwards. Though the expected true two-particle equilibrium distribution

$$
f_{0}(x, y)=\alpha^{2} \exp [-\alpha(x+y)]
$$

is certainly a fixed point of the transformation (4), this evolution alone cannot explain relaxation to (5) from arbitrary initial $f(x, y)$ because this requires new random pairings of particles at each step, not included in (41). That is why (4) has a lot of additional spurious 'equilibriums' - any function that depends only on $x+y$ does not change under this transformation. For these reasons only one iteration of Eq. (4) really makes sense and its only purpose is to produce nonlinear equation (1) after some 'projection' procedure, described below.

But let us first show that entropy grows for the transformation (4). The regular way to prove such monotonicity theorems is to start from the relative entropy or Kullback-Leibler (KL) distance $D(\mu \| \nu)=\sum \mu \ln \mu / \nu$ between two probability distributions $\mu$ and $\nu$. It is well known from information theory that $D(\mu \| \nu)$ cannot increase under 'coarse-graining' of these distributions, when some variables are integrated out. This immediately follows from the chain rule for relative entropy 11] and some examples of how this works may be found e.g. in $[12,13]$. Consider now the distribution

$$
\mu(\xi, x, y)=f(\xi(x+y),(1-\xi)(x+y)),
$$

defined on the space $\xi \in[0,1], x, y \in[0, \infty)$ and define $\nu(\xi, x, y)$ in the same way through the equilibrium distribution $f_{0}(x, y)$ from (5). It is easy to check that both $\mu$ and $\nu$ are positive and normalized to unity. Then define the coarse-graining procedure $\mu \rightarrow \tilde{\mu}$ as averaging over the $\xi$ variable, i.e.

$$
\tilde{\mu}(x, y)=\int_{0}^{1} d \xi \mu(\xi, x, y)=f^{\prime}(x, y),
$$

according to Eq. (4), and, obviously, $\tilde{\nu}(x, y)=f_{0}(x, y)$.

The above statement about the monotonic behavior of KL distance can be written as

$$
\begin{array}{r}
\int_{0}^{1} d \xi \int_{0}^{\infty} d x d y \mu(\xi, x, y) \ln \frac{\mu(\xi, x, y)}{\nu(\xi, x, y)} \geq \\
\geq \int_{0}^{\infty} d x d y \tilde{\mu}(x, y) \ln \frac{\tilde{\mu}(x, y)}{\tilde{\nu}(x, y)}
\end{array}
$$

In the integral on the left-hand side we now make a change of variables

$$
u=\xi(x+y), \quad v=(1-\xi)(x+y), \quad z=x-y
$$


with the obvious property $x+y=u+v$. Then the integration measure and ranges of integration transform as follows

$$
\int_{0}^{1} d \xi \int_{0}^{\infty} d x d y=\frac{1}{2} \int_{0}^{\infty} d u d v \int_{-(u+v)}^{(u+v)} d z \frac{1}{u+v}
$$

and since according to (6) the integrand does not depend on $z$, integration over $z$ exactly cancels the Jacobian $1 / 2(u+v)$.

Then, using also exact expressions for $\tilde{\mu}$, Eq. (7), and $\tilde{\nu}$, we can rewrite Eq. (8) as

$$
\begin{aligned}
& \int_{0}^{\infty} d u d v f(u, v) \ln \frac{f(u, v)}{f_{0}(u, v)} \geq \\
& \geq \int_{0}^{\infty} d x d y f^{\prime}(x, y) \ln \frac{f^{\prime}(x, y)}{f_{0}(x, y)}
\end{aligned}
$$

Thus the relative entropy could not increase under the transformation (4). Certainly the same inequality will be valid if we substitute any normalized fixed point solution of (44) instead of the exponential distribution $f_{0}(x, y)$, but choosing $f_{0}$ is more suitable for what follows.

The same way of reasoning may be applied actually for any linear Markov evolution of the form (2) with $\mathrm{P}(x, u)$ independent of $p(x)$. One should take $\mu(x, u)=\mathrm{P}(x, u) p(u), \nu(x, u)=\mathrm{P}(x, u) p_{0}(u)$, where $p_{0}(u)$ is an equilibrium distribution, and then integrate them over $u$ to obtain $\tilde{\mu}=p^{\prime}(x)$ and $\tilde{\nu}=p_{0}(x)$. Then from equation similar to Eq. (8) it follows that $K=\int d x p(x) \ln p(x) / p_{0}(x)$ monotonically decreases on each iteration, which is, of course, well known. The main idea behind this proof is that any such evolution may be viewed as some kind of coarse-graining since it includes integration over initial data (cf. [14]). Note that for nonlinear evolution, when $\mathrm{P}(x, u ; p)$ depends on the distribution function $p(u)$, as in (2), and hence changes on each step, this approach does not work, because now $p_{0}$ is not an instantaneous equilibrium and $\tilde{\nu} \neq p_{0}(x)$.

Now, since we have chosen the equilibrium distribution in the exponential form, $f_{0} \sim \exp [-\alpha(x+y)]$, Eq. (11) may be rewritten, as usual, as $F \geq F^{\prime}$, where $F=\langle x+y\rangle-S / \alpha$ is the free energy and the entropy is given by $S(f)=-\int d x d y f(x, y) \ln f(x, y)$. But our linear transformation conserves the mean energy $\langle x+y\rangle$, hence the monotonicity of the free energy results in the entropy growth

$$
S^{\prime} \geq S
$$

Thus we have proved the monotonic entropy growth for our auxiliary linear transformation (4). This is an almost evident result and it is only the first step of the proof for the original nonlinear problem.

Now we need to relate the linear process (4) to the initial nonlinear evolution (1). For this purpose consider in Eq. (4) a special factorized initial condition

$$
f(x, y)=p(x) p(y)
$$

and define the transformed probability $p^{\prime}(x)$ as the marginal probability for the transformed distribution, i.e.

$$
p^{\prime}(x) \equiv \int_{0}^{\infty} d y f^{\prime}(x, y) .
$$

Since $f^{\prime}(x, y)$ is symmetric under the permutation of variables $x$ and $y$ it actually does not matter which one variable to integrate out.

Then we have

$$
\begin{aligned}
p^{\prime}(x)= & \int_{0}^{\infty} d y \int_{0}^{1} d \xi p(\xi(x+y)) p((1-\xi)(x+y))= \\
& =\int_{0}^{\infty} d u \int_{0}^{\infty} d v \frac{\theta(u+v-x)}{u+v} p(u) p(v),
\end{aligned}
$$

where we have made the change of variables $(\xi, y) \rightarrow$ $(u, v)$ similar to (9), $u=\xi(x+y), v=(1-\xi)(x+y)$, with the Jacobian $1 /(u+v)$. The condition $u+v>x$ arises from the positivity of $y=u+v-x$. Clearly this is exactly the required nonlinear Eq. (1).

Thus, on each step the nonlinear evolution of the gaslike model may be obtained by a kind of 'projection' procedure from the linear transformation (4) by choosing the special initial conditions (13) and by the subsequent elimination of one variable from the resulting two-particle distribution function (14).

Another way to look at this phenomenon is to say that our non-linear evolution may be represented as a combination of two processes. The first one is the linear evolution of Eq. (4) with initial condition (13) which should be supplemented then by the subsequent 'reduction' of $f^{\prime}(x, y)$ back to the factorized form, which corresponds to a new random pairing of particles and is, in its turn, the new initial condition for the next step.

But elimination of exactly half of the variables, as in Eq. (14), is also related to some monotonicity property. For example, for the real space decimation renormalization transformation in spin systems, when on each step of renormalization we divide the lattice into two identical sublattices and half of all spins are summed away [15], the entropy per lattice site was shown to grow monotonically [13. For the sake of completeness we repeat here this simple derivation as applied to our present system.

This monotonicity of entropy per degree of freedom results just from the positivity of the mutual information of two sets of variables. In our present case the mutual information of $x$ and $y$ variables after the transformation (41), whose joint probability distribution is $f^{\prime}(x, y)$ and marginal distributions are $p^{\prime}(x)$ and $p^{\prime}(y)$, is given by the usual formula [11]

$$
I=\iint d x d y f^{\prime}(x, y) \ln \frac{f^{\prime}(x, y)}{p^{\prime}(x) p^{\prime}(y)} \geq 0 .
$$

This mutual information may be written also as a difference between the sum of entropies of subsystems (which are identical in our case) and the total entropy of the joint distribution

$$
I=2 S\left(p^{\prime}\right)-S\left(f^{\prime}\right)
$$


where $S\left(p^{\prime}\right)=-\int d x p^{\prime}(x) \ln p^{\prime}(x)$ and $S\left(f^{\prime}\right)$ is the entropy of the two particle system which earlier in Eq. (12) was denoted by $S^{\prime}$.

Hence from $I \geq 0$ it follows

$$
S\left(p^{\prime}\right) \geq \frac{1}{2} S^{\prime} .
$$

Note that this inequality is not just a trivial consequence of the information loss or decrease of the relative entropy after one variable is eliminated. Information loss results in the decrease of the total entropy, which looks like $S^{\prime} \geq$ $S\left(p^{\prime}\right)$ [13] and is clearly distinct from Eq. (18).

Now we can combine this inequality with the one obtained earlier for the linear evolution, Eq. (12), to arrive at $S\left(p^{\prime}\right) \geq S / 2$. But for the factorized initial distribution Eq. (13) the entropy $S$ is just twice the one-particle entropy of the distribution $p(x)$, i.e. $S=2 S(p)$ and hence we finally have

$$
S\left(p^{\prime}\right) \geq S(p) .
$$

This completes the proof that the entropy $S(p)=$ $-\int d x p(x) \ln p(x)$ monotonically grows on each step under iterations of the nonlinear transformation (11).

Let us now give an example illustrating our general proof. If we start from the distribution $p(x)=x \exp (-x)$ with entropy $S(p)=\gamma+1 \simeq 1.5772(\gamma \simeq 0.5772$ is the Euler constant), then for the factorized initial condition (13) we have $f^{\prime}(x, y)=1 / 6(x+y) \exp [-(x+y)]$ from Eq. (44). The corresponding entropy per degree of freedom is now larger, $S^{\prime} / 2=\gamma+1 / 6+1 / 2 \ln (6) \simeq 1.6397>S(p)$. After we eliminate one variable we finally have $p^{\prime}(x)=$
$1 / 6\left(x^{2}+2 x+2\right) \exp (-x)$ (see also [5]) and the entropy now equals $S\left(p^{\prime}\right) \simeq 1.6667$ which in its turn is slightly larger than $S^{\prime} / 2$. Thus we see how entropy indeed grows on each stage of our combined evolution that is equivalent to the initial nonlinear transformation.

In summary, we have proved the monotonic entropy growth for a nonlinear evolution which describes pairwise interaction of economical agents with random money exchanges and also may be viewed as a particular case of Ulam's redistribution of energy problem. The proof is based on representing a single step of the nonlinear evolution as a combination of two steps: The first is related to an auxiliary linear two-particle process and the second one is a kind of a coarse-graining, similar to decimation renormalization transformation, when one of the two variables is integrated away. Since on both steps the entropy can be shown to increase we conclude that the entropy is indeed monotonically increasing for the original nonlinear problem.

The proof is based entirely on information theory inequalities and possibly may be of some use for other nonlinear problems. It is not clear however whether it is possible to use the present approach or some of its modifications to find Lyapunov functions for non-uniform redistribution laws in the general Ulam problem.

I am very grateful to J. Gaite for pointing out Ref. 7] and valuable comments, to R. López-Ruiz for stimulating correspondence and important remarks, to V. Losyakov for many discussions and to J.L. McCauley for sending me his papers. The work was supported in part by RFBR Grants No. 10-02-00509, 11-02-90453 and 12-02-00520.
[1] J. Schnakenberg, Rev. Mod. Phys. 48571 (1976);

[2] F. Schlögl, Z. für Physik, 198559 (1967); F. Schlögl, Annals of Physics 45155 (1967); F. Schlögl, Z. für Physik, 243303 (1971);

[3] M. Esposito and C. Van den Broeck, Phys. Rev. E 82, 011143 (2010); H. Ge, and H. Qian, Phys. Rev. E, 81 051133 (2010); C. Maes, K. Netočný, and B. Wynants, Phys. Rev. Lett. 107010601 (2011);

[4] E.M. Lifshitz and L.P. Pitaevskii, Physical Kinetics (Butterworth-Heinemann Ltd, Oxford, 1999);

[5] R. López-Ruiz, arXiv:1009.3550; R. López-Ruiz, J.-L. López, X. Calbet, ESAIM Proceedings, 36 189-196 (2012); J.-L. López, R. López-Ruiz and X. Calbet, Journal of Mathematical Analysis and Applications 386195 (2012); X. Calbet, J.L. López, and R. López-Ruiz, Phys. Rev. E 83036108 (2011); E. Shivanian and R. LópezRuiz, Physica A 391 2600-2607 (2012);

[6] V.M. Yakovenko, in Encyclopedia of Complexity and System Science, Meyers, R.A. (Ed.) (Springer, Germany, 2009); B.K. Chakrabarti, A. Chatterjee, A. Chakraborti and S. Sinha, Economics: An Introduction (Willey-VCH Verlag GmbH, Germany, 2010);
[7] D. Blackwell and R.D. Mauldin, Letters in Math. Phys. 10149 (1985);

[8] E.T. Jaynes, Papers on Probability, Statistics and Statistical Physics (Reidel, Dordrecht, 1983);

[9] T.D. Frank, Phys. Lett. A 3724553 (2008); T.D. Frank, J.Phys. A: Math. Theor. 41282001 (2008); V.N. Kolokoltsov, Nonlinear Markov processes and kinetic equations, (Cambridge University Press, Cambridge, 2010);

[10] J.L. McCauley, Physica A 382445 (2007); J.L. McCauley, Physics Procedia 31659 (2010);

[11] T.M. Cover and J.A. Thomas, Elements of Information Theory, 2nd ed. (Wiley, Hoboken, NY) 2006;

[12] A. Gomez-Marin, J. M. R. Parrondo, and C. Van den Broeck, Phys. Rev. E 78011107 (2008);

[13] S.M. Apenko, Physica A 39162 (2012);

[14] S.M. Apenko, AIP Conf. Proc. 1332259 (2011);

[15] L.P. Kadanoff and A. Houghton, Phys. Rev. B 11377 (1975); L.P. Kadanoff, Rev. Mod. Phys. 49267 (1967); B. Hu, Phys. Rep. 91233 (1982). 